\title{
A Case of ST-Elevation due to a Primitive Extracardiac Origin in a Cancer Patient: Differential Diagnosis and Review of Literature
}

\author{
Amabile Valotta, MD*, Lorenzo Grazioli Gauthier, MD, Laura Anna Leo, MD, Manuela \\ Averaimo, MD and Marco Moccetti, MD
}

Department of Cardiology, Cardio Centro Ticino, via Tesserete 48, Lugano 6900, Switzerland

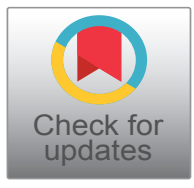

*Corresponding author: Amabile Valotta, Department of Cardiology, Cardiocentro Ticino, via Tesserete 48, Lugano 6900, Switzerland, E-mail: valotta.amabile@gmail.com

\begin{abstract}
Introduction: Vast of cases ST elevation is due to coronary occlusion. Metastatic disease has been identified as a rare case of Acute Myocardial Infarction or artery occlusion.

Case description: This case is about a 65-year-old man with a diagnose of stage IV Thymic Carcinoma in 2012, with two recurrences and past surgical treatment followed by radiation and chemotherapy. From that time, CPAP addicted for respiratory failure. He went to emergency department with worsening dyspnoea in the last few days. The ECG showed an ST-elevation in DI-aVL and V4-V6 suggesting an Acute Coronary Syndrome (ACS). The emergency coronary angiogram showed no significant coronary artery stenosis. The transthoracic echo revealed a mass infiltrating the left ventricular myocardium with consequent akinesia of lateral and anterior wall. A thoracic CT scan revealed a mass of $17^{*} 10^{*} 12 \mathrm{~cm}$ that infiltrated the myocardium surrounding the left circumflex coronary artery, which was the reason of ST-elevation.

Discussion: After multidisciplinary medical evaluation, considering the clinical context and the surgical risk, we dropped to perform any other invasive or bioptic exam. In agreement with the patient and his relatives, the patient was hospitalized Palliative Care Unit.

Conclusion: The ECG changes due to metastatic lesions or myocardial infiltration are different from those of an ACS. They are characterized by the persistence of ST-elevation without the typical evolution such as $Q$ waves or $T$ inversion. In addition, in any patient with history of malignancies, multidisciplinary discussion and multimodality imaging play a key role for the correct diagnosis and management.
\end{abstract}

\section{Keywords}

Cancer, Computed tomography, Echocardiography, Imaging, Palliative care

\begin{abstract}
Abbreviations
ACS: Acute Coronary Syndrome; AMI: Acute Myocardial Infarction; CPAP: Continuous Positive Airway Pressure; CT: Computed Tomography; IVC: Inferior Vena Cava; TnThs: High Sensitive T Troponine; VMAT: Volumetric Modulated Arc Therapy
\end{abstract}

\section{Introduction}

Virtually, all cases of ST elevation are due to coronary occlusion. Metastatic disease has been identified as a rare case of Acute Myocardial Infarction or artery occlusion [1-3]. Tumour compression of a coronary artery or its extension in the myocardium could also cause ST elevation [4-6]. Many authors have reported persistent ST elevation in patients with malignancies with heart metastasis $[7,8]$. The most common cancers that give myocardial involvement are of lung, breast, oesophagus cancer, melanoma and lymphoma [9]. Generally, cardiac metastases are considered rare. The incidence of cardiac metastases reported in literature is variable from $2.3 \%$ and $18.3 \%$ [10-12].

As shown in Table 1, ST elevation could be caused by different clinical extracardiac conditions.

In cancer patients presenting ST elevation, it is nec-

Citation: Valotta A, Grazioli Gauthier L, Leo LA, Averaimo M, Moccetti M (2020) A Case of ST-Elevation due to a Primitive Extracardiac Origin in a Cancer Patient: Differential Diagnosis and Review of Literature. Int J Clin Cardiol 7:205. doi.org/10.23937/2378-2951/1410205

Accepted: December 03, 2020: Published: December 05, 2020

Copyright: (c) 2020 Valotta A, et al. This is an open-access article distributed under the terms of the Creative Commons Attribution License, which permits unrestricted use, distribution, and reproduction in any medium, provided the original author and source are credited. 
Table 1: ST-elevation, differential diagnosis [13].

\section{Extracardiac ST elevation: Differential diagnosis}

\begin{tabular}{|l|l|}
\hline Vascular & Aortic dissection, pulmonary thromboembolism \\
\hline Pulmonary & Pneumonia, COPD, mediastinal tumor \\
\hline Abdominal & Cholecystitis, pancreatitis, hiatal hernia, subdiaphragmatic abscess, peritonitis \\
\hline Oncologic & Metastases, Compression, Infiltration \\
\hline Electrolytes & Hyperkalemia, hyper/hypophosphatemia \\
\hline
\end{tabular}

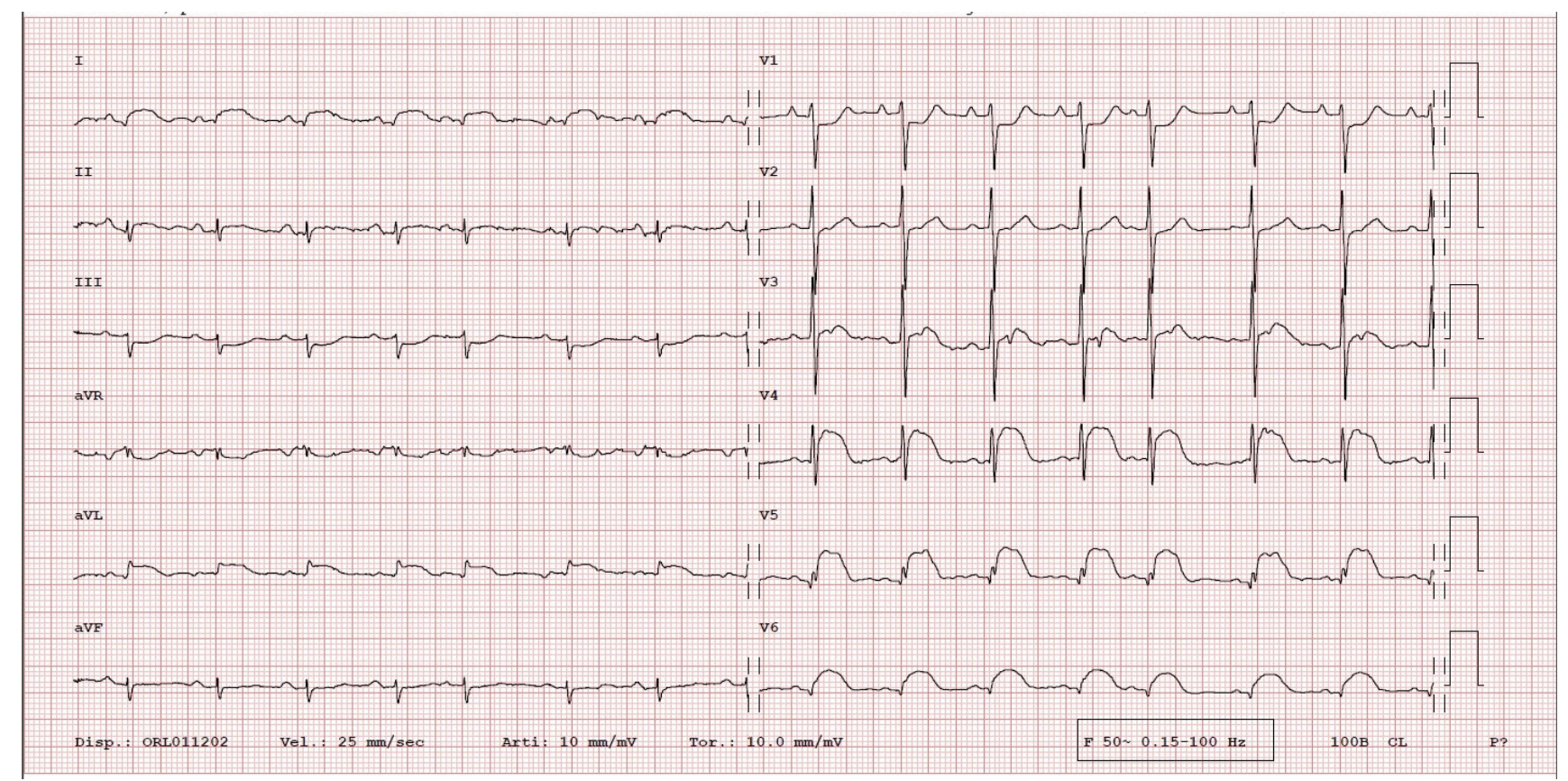

Figure 1: ECG: ST-elevation suggesting an ACS.

essary to consider differential diagnosis, first an acute coronary syndrome due to the typical pro-coagulant and inflammatory condition; in addition, in literature are reported cases of myopericarditis after immunotherapy [14], or Takotsubo syndrome related to physiologic or emotional stress and chemotherapy [15].

This case is about a patient with a stage IV Thymic Carcinoma that has infiltrated the myocardium surrounding the left circumflex coronary artery and has caused ST elevation.

\section{Timeline}

In December 2011, during investigations for recurrent pericarditis, a Thymic Carcinoma at stage IV was accidentally diagnosed; from January 2012 until February 2012, a Neoadjuvant chemotherapy was performed. In June 2012, the patient has undergone surgical treatment. In June 2013 there was the first cancer recurrence treated with chemotherapy (Carboplatin and Taxol). From October 2013 until May 2014 an experimental protocol with PHA 848125AC was performed and, after that, chemotherapy with Temozolomide was given. In November 2014, a laser treatment of bronchial obstruction was performed with positioning of tracheobronchial prosthesis; in December 2014 a radiotherapy of mediastinal lesion and right axillary lesion was necessary. In
April 2015, another radiotherapy (VMAT) on upper left pulmonary nodule was necessary. In August 2015, there was a second recurrence of cancer with right bronchial stenosis causing dyspnoea. From February 2017 until January 2018, an experimental protocol with Pembrolizumab was performed; in addition, in April 2017 a radiotherapy on a sternal injury was done. Finally, in January 2018 the patient started a CPAP therapy.

\section{Case Report}

This case is about a 65-year-old man who was diagnosed of stage IV Thymic Carcinoma in 2012, with two recurrences in 2013 and 2015. He underwent a surgical treatment in 2012 with surgical excision of the mediastinal mass, right upper and medium lung lobe, and upper left lobe, pericardiectomy, excision of phrenic nerve, IVC, innominate left vein and portion of innominate right vein, followed by different radiation and chemotherapy. From 2018, the patient was CPAP addicted for respiratory failure. At the time of our meeting with the patient, an experimental protocol with Pembrolizumab was still in progress. He was admitted to emergency department in a cachectic status with worsening dyspnoea in the last few days. The Glasgow Coma Scale was 15/15; the blood pressure was $90 / 60 \mathrm{mmHg}$ and the pulse rate 74 $\mathrm{bpm}$. The auscultation was totally subverted with harsh 
lung sound, heart tones were difficult to hear. The biochemistry panel revealed TnT hs $35 \mathrm{ng} / \mathrm{L}, \mathrm{CRP} 80 \mathrm{mg} / \mathrm{L}$, creatinine $80 \mathrm{umol} / \mathrm{L}$, GFR $90 \mathrm{ml} / \mathrm{min} / 1.73 \mathrm{~m}^{2}$. He denied any chest pain, and he complained of dyspnoea and weakness. The ECG showed an ST-elevation in DI-aVL and V4-V6 suggesting an ACS (Figure 1), so the patient has been urgently transferred to hemodynamic units.
An emergency coronary angiogram was performed but the exam showed no significant coronary artery stenosis (Figure 2). The transthoracic echocardiography performed in the cath lab revealed a mass infiltrating the left ventricular myocardium with consequent akinesia of lateral and anterior wall without impairment of the right ventricle or valvular disease (Figure 3). For a bet-

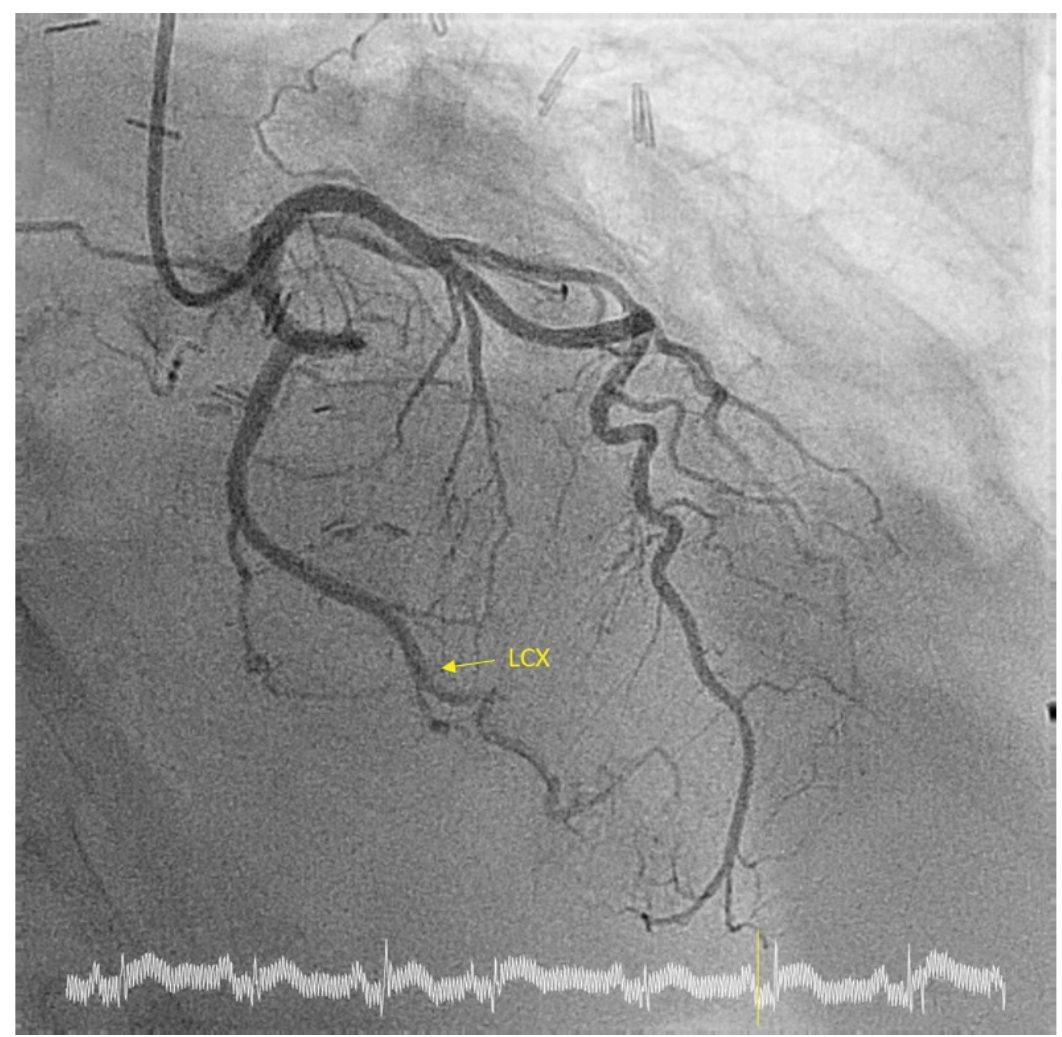

Figure 2: Coronary Angiogram: Absence of significant coronary artery stenosis.

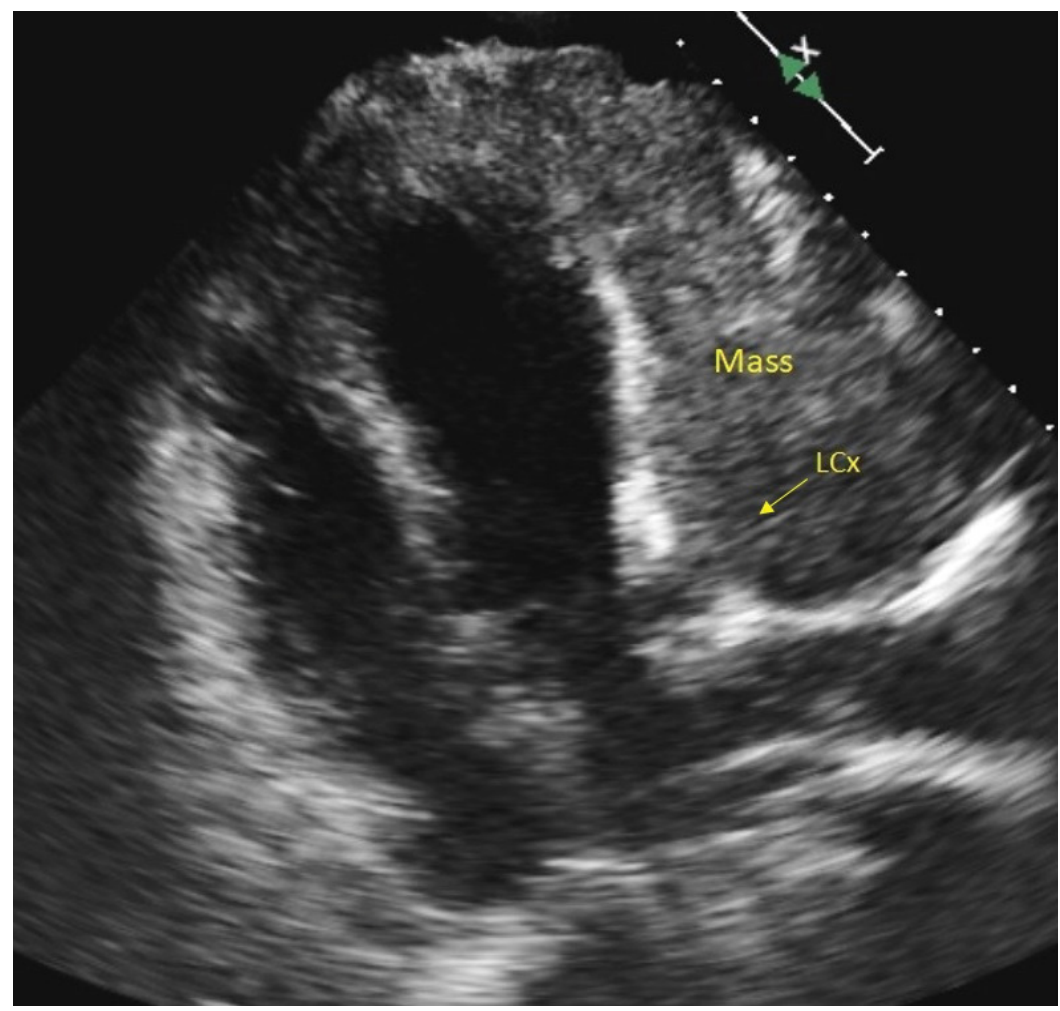

Figure 3: Transthoracic Echocardiography: Mass infiltrating the left ventricular myocardium. 


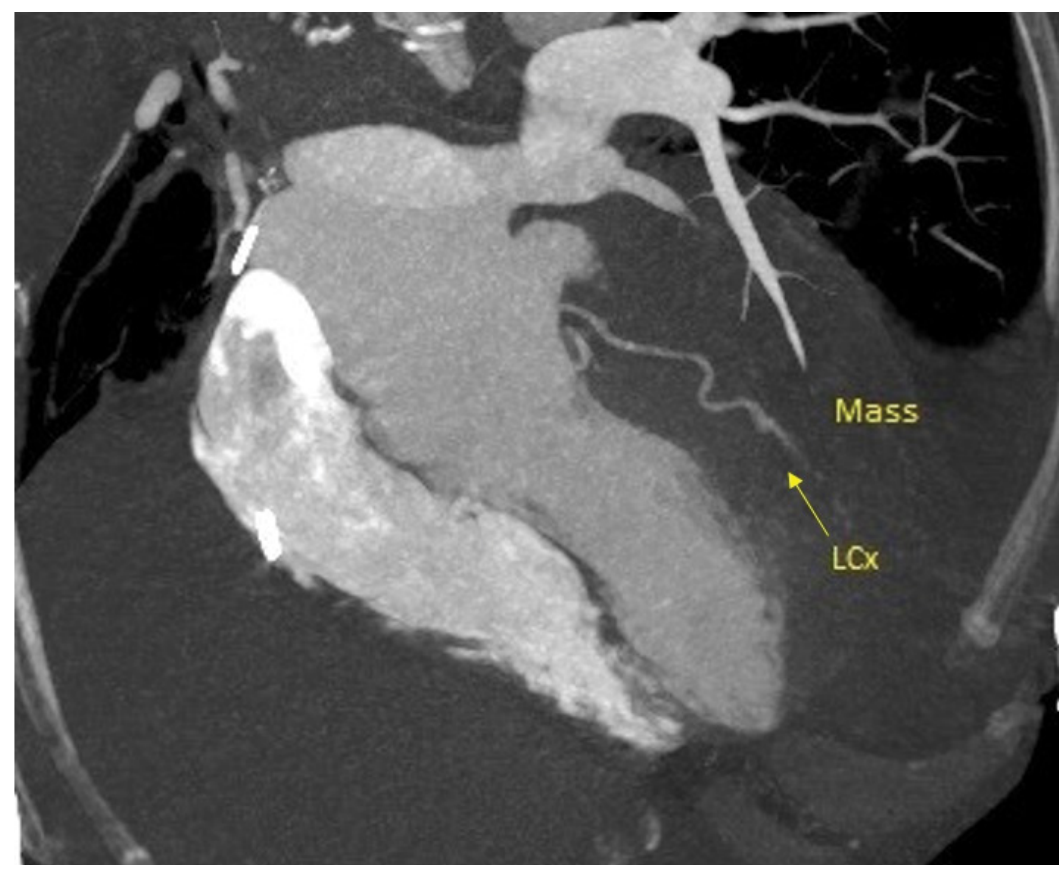

Figure 4: CT scan: Mass without a cleavage plan infiltrating the myocardium.

ter diagnostic picture, we performed a thoracic CT scan (Figure 4). The exam revealed a mass of $17^{*} 10^{*} 12 \mathrm{~cm}$ without a cleavage plan from the LV, which infiltrated the myocardium surrounding the left circumflex coronary artery. This finding was interpreted as the cause of the ST-elevation.

\section{Discussion}

After multidisciplinary medical evaluation, considering the clinical context and the prohibitive surgical risk, we dropped to perform any other invasive or bioptic exam. In agreement with the patient and his relatives, a symptomatic and supportive therapy was only considered, so the patient was hospitalized in Palliative Care Unit. Unfortunately, the patient died a few days later for respiratory distress.

\section{Conclusion}

Patients with history of cancer may have symptoms and ECG changes mimicking ACS [3]. The ECG changes due to metastatic lesions or myocardial infiltration are different from those of an ACS [16]; they are characterized by the persistence of ST-elevation without the typical evolution such as $Q$ waves or $T$ inversion. In addition, alterations of ventricular repolarization are a distinctive marker of cardiac metastasis [17]. The presence of cardiac metastasis is often an accidental diagnosis or it occursat the appearance of systemic symptoms [12]; it is also possible that cardiac lesions remain clinically silent and discovered at autopsy [18]. As acknowledged, patients with secondary cardiac involvement have a poor prognosis [3]. The role of radio- or chemotherapy remains unclear for the lack of evidence; palliative therapy remains the most widely used therapeutic strategy [4].

In our case, the patient complains of dyspnoea, the
ST-elevation did not evolve as a typical ACS. The clinical presentation and the medical history of the patient could drive to the correct differential diagnosis.

Finally, in any patient with history of malignancies, multidisciplinary discussion and multimodality imaging play a key role for the correct diagnosis and management.

\section{Authors Declaration}

All authors have participated in the work, have reviewed, and agree with the content of the article.

\section{References}

1. Franciosa JA, Lawrinson W (1971) Coronary artery occlusion due to neoplasm: A rare cause of acute myocardial infarction. Arch Intern Med 128: 797-801.

2. Lam KY, Dickens $P$, Chan AC (1993) Tumors of the heart. A 20-year experience with a review of 12,485 consecutive autopsies. Arch Pathol Lab Med 117: 1027-1031.

3. Suga T, Akuzawa N, Hatori T, Imai K, Kitahara Y, et al. (2015) ST elevation in secondary cardiac cancer: A case report and review of the literature. Int J Clin Exp Med 8: 7719-7727.

4. Patel H, Francke M, Stahura H, El-Hajjar M, SchulmanMarcus J (2018) Solitary cardiac metastasis from primary oral squamous cell carcinoma presenting as ST-elevation MI. BMJ Case Rep.

5. Pan KL, Wu LS, Chung CM, Chang ST, Lin Pc, et al. (2007) Misdiagnosis: Cardiac metastasis presented as a pseudoinfarction on electrocardiography. Int Heart J 48: 399-405.

6. Weinberg BA, Pinkerton CA, Waller BF (1990) External compression by metastatic squamous cell carcinoma: $A$ rare cause of left main coronary artery narrowing. Clin Cardiol 13: 360-366.

7. Rodrigues AC, Abreu E, Demarchi LM, Wilson MJr, Samira Leal MB, et al. (2003) Lung neoplasm mimicking an acute lateral myocardial infarction. J Am Soc Echocardiogr 16: 1198-1200. 
8. Astorri E, Fiorina P, Pattoneri P, Paganelli C (2001) Persistent ST segment elevation in a patient with metastatic involvement of the heart. Minerva Cardioangiol 49: 81-85.

9. Tandon V, Kethireddy N, Balakumaran K, Kim SA (2019) Metastatic squamous cell carcinoma to the heart: An unusual cause of ST elevation-a case report. Eur Heart JCase Rep 3: 1-7.

10. Butany J, Leong SW, Carmichael K, Komeda M (2005) A 30 -year analysis of cardiac neoplasms at autopsy. Can J Cardiol 21: 675-680.

11. Klatt EC, Heitz DR (1990) Cardiac metastases. Cancer 65: 1456-1459.

12. Tyebally S, Chen D, Bhattacharyya S, Mughrabi M, Hussain Z, et al. (2020) Cardiac Tumors: JACC CardioOncology State-of-the-Art Review. JACC: CardioOncology 2: 293-311.
13. Sethi P, Murtaza G, Sharma A, Paul T (2016) ST segment elevation with normal coronaries. Case Rep Med 2016: 3132654

14. Heinzerling L, Ott PA, Hodi FS, Husain AN, Tajmir-Rihai A, et al. (2016) Cardiotoxicity associated with CTLA4 and PD1 blocking immunotherapy. J Immunother Cancer 4: 50.

15. Coen M, Rigamonti F, Roth A, Koessler T (2017) Chemotherapy-induced Takotsubo cardiomyopathy, a case report and review of the literature. BMC Cancer 17: 394.

16. Cates CU, Virmani R, Vaughn WK, Robertson RM (1986) Electrocardiographic markers of cardiac metastasis. Am Heart J 112: 1297-1303.

17. Bussani R, De Giorgio F, Abbate A, Silvestri F (2007) Cardiac metastases. J Clin Pathol 60: 27-34.

18. Samaras P, Stenner-Liwen F, Bauer S, Goerres GW, von Boehmer L, et al. (2007) Infarction-like electrocardiographic changes due to a myocardial metastasis from a primary lung cancer. Circulation 115: e320-e321. 